\title{
Enfrentando el desafío del alto corte agua en campos petroleros: causas, diagnóstico y mecanismo químico de control
}

\author{
Anderson Mosquera Ruiz ${ }^{1 *}$
}

doi: http://dx.doi.org/10.18273/revfue.v18n2-2020006 (c) (1)

Forma de citar: Mosquera-Ruiz, A. (2020). Enfrentando el desafío del alto corte agua en campos petroleros: causas, diagnóstico y mecanismo químico de control. Fuentes, el reventón energético, 18(2), 89-105. https://doi.org/10.18273/revfue.v18n2-2020006

\section{Resumen}

El agua producida de los yacimientos petroleros representa un reto medioambiental, técnico y económico que debe ser resuelto para obtener operaciones más rentables, sostenibles, ininterrumpidas y amigables con el medioambiente. Por medio de este trabajo se exploran y caracterizan los principales temas en torno al problema de la excesiva producción de agua, entre los cuales se encuentran áreas críticas como indicadores de un alto corte de agua, los mecanismos para determinar la existencia de una producción excesiva de agua, algunos de los medios disponibles para resolver la problemática y se da un énfasis especial al control de agua mediante geles de reducción de permeabilidad relativa. Para esto se realizó una revisión bibliográfica, donde se llevó a cabo una selección y clasificación por categorías del tema de estudio. En este artículo se abordan cronológicamente los principales temas de utilidad en el control de agua y se pueden identificar algunos vacíos, conceptos que los investigadores manejan de formas similares, y se puede ver cómo ha cambiado el paradigma del control de agua lo largo de los años. El principal aporte de este trabajo se centra en el resumen y organización cronológica de los principales temas en torno al problema de la excesiva producción de agua de los yacimientos petroleros y del uso de sistemas gelificantes para su control.

Palabras clave: producción de agua, geles entrecruzados, agua, diagnostico, RDP, control de agua, medio poroso.

\section{Facing the challenge of high water cut in oilfields: casues, diagnosis and chemical means of control}

\begin{abstract}
The water produced from oil fields represents an environmental, technical, and economic challenge that must be resolved in order to obtain more profitable, sustainable, uninterrupted and environmentally friendly operations. This paper explores and characterizes the main issues surrounding the problem of excessive water production, among which are critical areas such as indicators of a high water cut, mechanisms to determine the existence of excessive water production, some of the means available to solve the problem and there is a special emphasis on water control through gels that reduce the water relative permeability. For this purpose, a thorough bibliographic review was carried out, where a selection and classification by categories of the subject of study was made by the author. In this article, the main useful topics in water control are addressed chronologically and some gaps can be identified, some concepts that the researchers handle in similar ways and it is easy to see how the paradigm of water control has changed over the years. The main contribution of this work is centered in the summary and chronological organization of the main issues around the problem of excessive water production from oil fields and the use of gelling systems for its control.
\end{abstract}

keywords: water production, crosslinked gels, water, diagnosis, DPR, conformance control, porous media.

\footnotetext{
${ }^{1}$ Universidad Industrial de Santander, mosqueragidelpa@gmail.com
} 


\section{Introduccion}

La producción de agua es un problema técnico y financiero que obliga a las compañías productoras a cerrar pozos por los altos costos que implica mantenerlos activos. El hecho de realizar una inversión de alrededor de 2.6 Millones de dólares en promedio en la perforación de un pozo (Montes A., Carreño W. y Guio M., 2016, p. 3), y que al poco tiempo esta inversión se vea contrarrestada por problemas técnicos (Eoff L., Dalrymple D., Everett D. y Vásquez J., 2006), ha hecho que muchos investigadores busquen soluciones que ayuden a mantener los pozos abiertos a producción (Ortega A. T., Marín D. F. y Ochoa E. D., 2019); existen en el mercado mecanismos como los dispositivos de control de influjo (ICD por sus siglas en inglés) que seleccionan el tipo de fluido que ingresa a un pozo productor a través de las perforaciones (Daneshy A.A., Krasnov V.A. y Zimin S.V., 2010, p. 3 ); otra de las soluciones, son los polímeros y los sistemas gelificantes conocidos también como fluidos de reducción desproporcionada de la permeabilidad (DPR por sus siglas en inglés) (Erazo-Bone R., Escobar-Segovia K., España F. y Hernández T., 2019), que se posicionan en el espacio poroso permitiendo que el aceite fluya con normalidad y que el agua sea retenida en el yacimiento (Tapias F.A., Lizcano J.C. y Lopes R.B.Z., 2018, p. 2).

Antes de intentar solucionar un problema lo primero es identificar su causa, para ello existen algunas técnicas que, a través de las herramientas e información disponible de un pozo (Busaidi K. y Bhaskaran H., 2003), permiten conocer la existencia de un problema de producción de agua, el punto donde se genera la problemática y el tipo específico de problema, ya que, no toda el agua que acompaña la producción de petróleo es obtenida de la misma manera y por lo tanto el mecanismo para controlarla variará (Alzate D., León. J. M., Cabrera F. y Manrique E., 2017); uno de los casos más comunes es cuando se inyecta agua al yacimiento con fines de mantenimiento de presión o recobro mejorado (Paris de Ferrer M., 2001), pero el agua inyectada no logra recuperar tanto petróleo como se esperó, se observa un problema de producción excesiva de agua que puede estar provocado por fracturas y fisuras en el subsuelo o por canales de alta permeabilidad entre los pozos inyector y productor.

Una de las bondades de las soluciones gelificantes como mecanismo para controlar la excesiva producción de agua, está en que pueden ser usadas para sellar los canales de alta permeabilidad, o pueden reducir dramáticamente la permeabilidad relativa al agua (Romero J. L., 2018).

En este artículo se realiza una revisión de las causas del exceso de la producción de agua, los métodos que existen para controlarla, se explica cómo están formados los sistemas gelificantes, qué efectos producen en el yacimiento y qué factores varían su efectividad.

\section{Líneas tematicas}

\section{Causas de la producción de agua}

En general, un problema de producción de agua puede ser muy complejo como para diferenciarlo en mecanismos específicos, pero diferentes autores han realizado clasificaciones con base en sus observaciones, se mencionarán aquí algunas de ellas:

Chan (1995) identificó tres problemas básicos: (1) conificación de agua, (2) canalización de varias capas y (3) problemas cerca del pozo (p. 1). Sparlin D.D. (1976) dividió el espectro de problemas en cuatro regímenes: el primero son problemas de fractura simples con o sin comunicación directa de fractura entre el pozo inyector y el productor; el segundo, corresponde a problemas de red de fracturas, en el que, fracturas por las cuales fluye solamente agua se conectan con fracturas a través de las cuales fluye algo de petróleo; El tercero tiene en cuenta estratos de diferente permeabilidad entre los cuales no existe el flujo cruzado; y el cuarto, el más difícil de solucionar (Aldhaheri M.N., Wie M., Bai B. y Alsaba M., 2016), a estratos de diferente permeabilidad donde sí existe el flujo cruzado (p. 5).

Posteriormente Syndask R.D. (1988) explicó que la “conificación es el proceso mediante el cual el gas o el agua libre es atraído a las perforaciones de producción en la zona petrolífera, en contra de las fuerzas de gravedad" (p. 3). Además de la conificación, el autor identificó la situación en la que existen dos capas adyacentes, una de las cuales produce solamente petróleo y la otra solamente agua; en esa situación observó que se pueden presentar dos casos: uno donde exista flujo cruzado entre las capas y otro donde no, siendo este último el caso más difícil de tratar usando soluciones gelificantes (Castro W.J. y Ordoñez D.F., 2019).

Zaitoun A., Kohler N., Bossie-Codreanu y Denys K. (1999) enuncian que la mayor parte de los fluidos producidos en campos maduros son agua debido a que el agua pasa a reemplazar los espacios vacíos 
que deja el petróleo en la matriz de roca cuando este último es producido. Además de lo anterior, exponen que muchos yacimientos son sometidos a procesos de recuperación secundaria mediante la inyección de agua lo que proporciona un mantenimiento en la presión de yacimiento, pero un aumento continuo en la producción de agua (Green D. W. y Willhite G. P., 1998); además de lo anterior identifican en su revisión la existencia de 8 problemas que causan la excesiva producción de agua: (1) fugas en el revestimiento, en la tubería de producción y demás tubulares del sistema de producción de petróleo; (2) flujo por detrás de la tubería de revestimientos; (3) yacimientos estratificados con barreras de flujo vertical; (4) fracturas individuales entre inyectores y productores; (5) conificación a través de fracturas; (6) canalización entre una red de fracturas naturales (7) formación de conos; (8) flujo sin barreras de flujo vertical. (Seright como fue citado en (Zaitoun A., Kohler N., Bossie-Codreanu y Denys K., 1999, p. 1).

En el 2000 Baily B. et al. presentaron al mundo un artículo (2000) en el cual clarifican muchos de los conceptos usados en el control de agua. Por ejemplo, se clasifica el agua en 3 tipos: (1) agua de barrido (de inyección o proveniente de un acuífero) la cual ayuda a barrer el aceite del yacimiento; (2) agua "buena" la cual es producida en tasas que son aceptables económicamente o sin la cual no podrían producirse aún pequeñas tasas de petróleo (p. 4); y finalmente, (3) agua "mala" la cual se produce a tasas tan altas en comparación con el petróleo que es mejor cerrar los pozos para evitar pérdidas por costos de operación, separación y tratamiento del agua. (p. 5).

En este artículo también se menciona que la producción de agua "mala" en pozos individuales puede clasificarse en 10 tipos básicos, los cuales serán mencionados a continuación (p. 5-7):

1. Fugas a través del casing, tubing o empaques

2. Canal de flujo detrás del casing

3. Contacto agua-aceite en movimiento

4. Estrato saturado de agua, sin movimiento vertical de fluido

5. Fracturas o fallas entre el pozo inyector y el pozo productor

6. Fracturas o fallas desde un estrato saturado de agua

7. Conificación o encrestamiento

8. Barrido areal deficiente (Vásquez J. y Rodríguez L., 2015)

9. Segregación gravitacional al interior de un estrato
10. Estrato saturado de agua, con movimiento vertical de fluidos.

Los aportes que presentan los investigadores a partir del año 2000 se enfocan en generar nuevas clasificaciones que permitan identificar rápidamente el problema y aplicar una solución para prevenir las pérdidas económicas (El-Karsani K.S.M., Al-Muntasheri G.A. y Hussein I.A., 2014):

Vásquez J. y Eoff L., dividen los casos problemáticos en 2: yacimientos estratificados (yacimientos con propiedades de porosidad y permeabilidad que varían entre estratos), con estratos que no estén comunicados entre sí $(2013$, p. 4), y yacimientos con flujo cruzado entre los estratos.

Luego Hatzignatiou D.G., Helleren J. y Stavland A., presentaron un artículo (2014) en el cual explican cuál es el mecanismo a través del cual el agua ocupa el espacio poroso y posteriormente irrumpe a los pozos productores. Dicen que el agua está presente de manera natural en los yacimientos que contienen hidrocarburos y que se distribuye de acuerdo a fuerzas gravitacionales (densidad) y fuerzas capilares (mojabilidad y tensiones interfaciales); durante la producción, las fuerzas viscosas son muy importantes ya que regulan la velocidad de movimiento de fluido para un nivel de energía particular (Paris de Ferrer M., 2009). Los autores exponen que a medida que el yacimiento se hace más maduro, la cantidad de agua producida aumenta y que al implementar técnicas de recobro mejorado como la inyección de agua y gas la producción de agua sigue aumentando (debido a las fuerzas viscosas), conectado a esto está el aumento en los costos de producción de hidrocarburos, la disminución en la tasa de aceite y la disminución en la capacidad de las facilidades de superficie para realizar la separación y el tratamiento de los fluidos producidos, factores que llevan a la exacerbación del problema económico para las compañías productoras. Una causa del aumento en los costos de producción de hidrocarburos es el aumento en los requerimientos de la regulación (ARPEL, s.f.), la cual exige que el agua debe ser tratada hasta cumplir valores específicos en parámetros como $\mathrm{pH}$, Temperatura y cantidad de materia orgánica disuelta antes de ser vertida o reinyectada (Presidencia de la Republica de Colombia, 2015).

Se tiene la evidencia de que Aldhaheri M.N., Wie M., Bai B. y Alsaba M. desarrollaron una nueva manera de clasificar los problemas de producción de agua (2015). 
Para ellos los problemas de producción de agua pueden ser explicados por varios factores de yacimiento como la dirección de la heterogeneidad en la permeabilidad, la presencia de flujo cruzado y el tipo de yacimiento (fracturado o de roca matriz).

Estos problemas, según los autores pueden ser agrupados en dos categorías dependiendo del grado de comunicación entre el pozo inyector y el productor:

1. Canalización directa: consiste en una fuerte y severa comunicación entre el pozo productor y el inyector.

2. Canalización indirecta: son identificados cuando la tasa de inyección es diferente a la tasa de producción de fluidos; para este caso se usa el término Flujo dominado por la matriz.

Estos pueden ser reclasificados dependiendo del patrón de comunicación en:

1. Limitadas: en los cuales la extensión de la zona productora de agua es muy pequeña.

2. Extensas: la zona para remediar es muy grande.

Posteriormente se puede clasificar el resultado de lo anterior dependiendo del estado de desarrollo del problema en:

1. Subdesarrollado: cuando el problema se presenta en las etapas tempranas de la inyección de agua.

2. Desarrollado: en la cual la saturación de aceite de la zona es baja porque el agua ha barrido el petróleo.

Para cerrar el análisis de las causas de la producción excesiva de agua, se observó que en el año 2014 se produjo un cambio en la organización de los problemas de producción de agua, dejando de publicarse clasificaciones en categorías lineales y pasando a una organización en forma matricial con base en las características comunes que compartían los problemas de producción de agua (si se producían al interior de la matriz o eran un problema del pozo, etc.) (Misha A., Abbas S., Braden J., Hazen M., Li G., Peirce J. y Smith D.D., 2016).

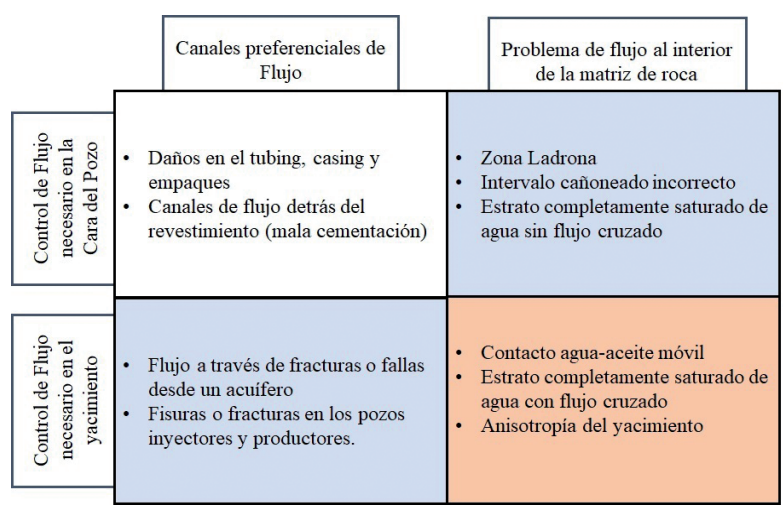

Figura 1. Matriz de diagnóstico de los problemas de producción de agua. (Modificado de Aldhaheri M. N., Wei M., Bai B., y Alsaba M., 2016)

\section{Diagnóstico}

K.S. Chan (1995) compartió un método para descubrir si el agua producida aportaba en algo al arrastre de petróleo o no; en la descripción de su método muestra que aproximarse a un gráfico convencional de corte de agua vs tiempo en una escala lineal no alcanza a ilustrar la diferencia existente entre la producción de fluidos desde múltiples capas y la conificación (p. 2), pero que al momento de graficar la relación agua aceite (WOR por sus siglas en inglés) vs el tiempo en un formato logarítmico se pueden observar las tendencias de producción y los problemas de producción de agua (Ramos L. y Marín A., 2018). Luego realizando una derivada de la gráfica anterior se puede diferenciar entre la conificación o el flujo vertical entre estratos. El autor observó que el valor inicial del WOR depende de la saturación inicial del yacimiento, la distribución de los fluidos en el espacio poroso y la permeabilidad de cada estrato; la duración de este periodo inicial, el cual termina en el momento en que el contacto agua aceite alcanza la perforación más profunda, se mide con respecto a la variación del WOR y depende del mecanismo de empuje el yacimiento, del movimiento del agua en el yacimiento, la distancia entre el contacto agua aceite (WOC por sus siglas en ingles) y la perforación más profunda, la relación entre la permeabilidad vertical y horizontal, la tasa de influjo desde el fondo, entre otros parámetros; para el caso en el que existe un problema por flujo cruzado entre estratos el final del periodo inicial está marcado por el momento en el que el agua irrumpe desde algún estrato a los pozos productores y lo importante aquí 
es la saturación inicial de fluidos, su distribución en el estrato y los contrastes de permeabilidad entre estratos. El autor identifica así mismo un segundo y tercer periodo en el cual el problema de producción de agua se hace más grave, evidenciándose con un aumento sostenido en el WOR debido al ascenso y crecimiento del cono (en el caso de conificación) y a la irrupción de agua (en el caso del flujo cruzado). El autor analiza que el cambio más considerable en cuanto a las tendencias en la producción de agua se da hacia periodos intermedios, observándose que en el caso de la conificación la producción de agua tarda mucho tiempo en aumentar, es decir, una vez que es alta hay un punto en el cual no aumenta tan rápido; en el caso del flujo cruzado entre estratos la producción de agua nunca deja de crecer, por lo cual la derivada del WOR con respecto al tiempo es una línea con pendiente positiva después de haberse dado el cambio del periodo de producción inicial en el cual la pendiente es cero (p. 6).
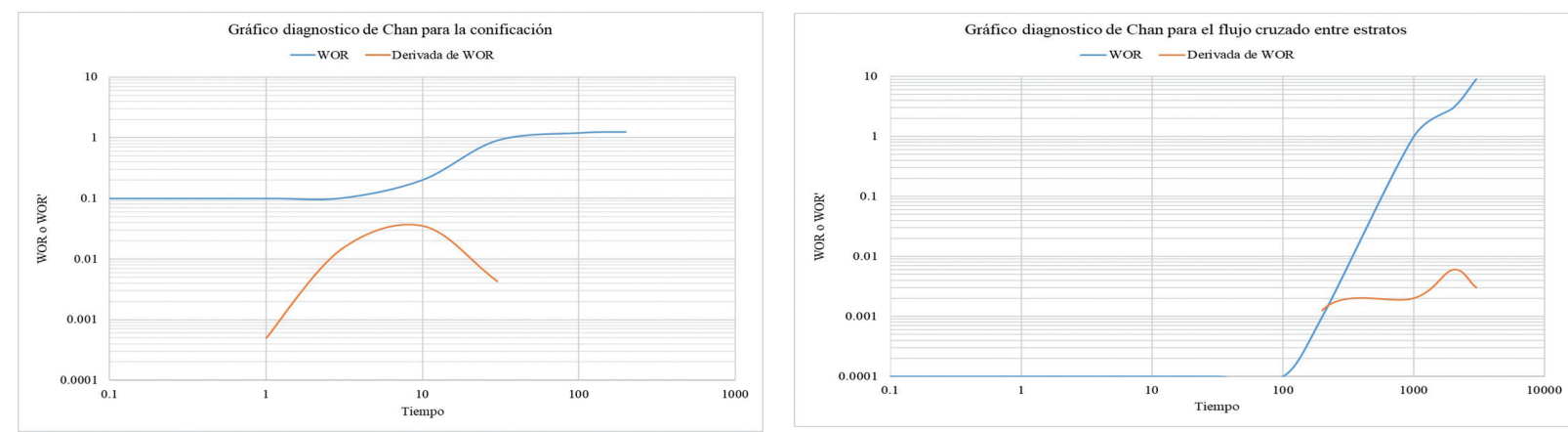

Figura 2. Gráfico diagnóstico de Chan para identificar la conificación o el flujo cruzado entre estratos. (Modificado de Baily B. et al. 2000).

Las observaciones realizadas en los cambios en la pendiente de la gráfica WOR vs tiempo, llevan a concluir que el análisis de una curva de la derivada del WOR vs tiempo puede ser útil para determinar el tipo de problema de producción de agua (p. 3). (Mejia H. D., 2016). Debido a todo lo anterior el investigador aconseja buscar el comportamiento normal de la producción (en términos de WOR), mirar la tendencia de su derivada y finalmente expandir los periodos de interés y observar los cambios con respecto al estado inicial (p. 5).

Baily B. et al (2000), enuncian que uno de los hechos que ha llevado a que sea difícil controlar la excesiva producción de agua es la falta de conocimiento y entendimiento de los diferentes problemas que existen y la consecuente aplicación indebida de los tratamientos. Por ello dicen que la clave para ser exitosos en el intento de controlar la producción de agua está en el diagnóstico, el cual puede ser usado de tres maneras: (1) para determinar qué pozos son candidatos a control de la producción de agua, (2) para determinar el problema de producción de agua y (3) para localizar el punto de entrada del agua al pozo (p. 8).

Se procederá a hacer un recuento de la información que puede ser usada para hacer un buen diagnóstico y de qué manera estas fuentes pueden ayudar en la confirmación de su existencia y la determinación del tipo de problema de producción de agua que se tiene (p. 8-14):

- Gráfico de Recuperación: muestra las tendencias de producción en el tiempo, donde puede evidenciarse si el agua ayuda o no a incrementar la producción de aceite.

- Gráfico de historia de producción: se ven directamente las tasas de producción de fluidos, de donde puede deducirse directamente si existe o no un problema de producción de agua.

- Curvas de declinación: se puede reconocer el mecanismo de empuje del yacimiento y si existe o no una depleción normal de acuerdo con los análisis realizados (Oghena A., 2012).

- Gráficos diagnósticos: describen la existencia de un problema de conificación o de flujo cruzado entre en estratos de la manera descrita por K.S. Chan (1995).

- Análisis Nodal: es usado para determinar el punto de entrada del agua en exceso, a través de balances energéticos sobre el sistema de producción.

- Registros de producción: se puede evidenciar directamente el punto de entrada del agua al sistema de producción a través del uso de sensores. 
Ortega A., en su tesis de grado (2013, p. 3043) argumenta que, para garantizar el éxito de la inyección de un tratamiento de fluidos de control de la producción de agua, se debe conocer muy bien la situación problema y que esto se logra a través de la adquisición de la mayor cantidad posible de información; en su revisión bibliográfica identifica algunos de los elementos enunciados por (Baily B. et al, 2000), aunque agrega a todos ellos lo siguiente:

- Registros de perforación: dentro de los cuales es importante revisar las zonas de perdida de circulación, ya que son canales de alta permeabilidad donde la integridad del cemento puede ser cuestionable.

- Registros de pozos: son usados para estimar las saturaciones, porosidad y permeabilidades de una zona, además de los registros a hueco revestido que sirven para tener alguna información de la efectividad de las operaciones realizadas durante el completamiento del pozo.

- Pruebas de Pozo: las cuales ayudan a conocer las condiciones de flujo del yacimiento y las propiedades petrofísicas que tiene cuando se somete a la producción de fluidos.

(Villegas I.A., 2013) у (Mendoza J.R., 2013) son autores que muestran la aplicación de proyectos de control de la producción de agua a nivel campo en Latinoamérica, entre sus mayores aportes se identifican la aplicación de técnicas de diagnóstico, selección de la solución apropiada y la aplicación de sistemas químicos para control de la producción de agua.

\section{Solución mecánica}

Las soluciones mecánicas son aquellas en las que se coloca en el pozo algún medio de contención de los fluidos de producción, como cemento (Borling D., Chan K., Hughes T. y Syndansk R.S., 1994, p. 3), empaques (Baily B. et al. 2000, p. 13), tuberías adicionales o los ICD los cuales modifican el patrón de influjo al pozo de los fluidos de yacimiento (Garzón M. F., Barrero L. P., Portilla H. E., Castillo G., 2014).

Este tipo de control de la producción de agua funciona cuando el agua se produce desde una zona que está completamente saturada de agua (Thomas F.B., Bennion D.B., Anderson G.E., Meldrum B.T. y Heaven W.J., 2000, p. 1); también debe tenerse en cuenta el tipo de completamiento, si es a hueco abierto, con empaque de grava y el diámetro interno de la tubería de completamiento, ya que la solución mecánica a implementar podría generar problemas de integrad en el pozo (Botermans C.W., D. W. van Batenburg y Bruining J., 2001, p. 1).

Según Taha A. y Amani M. algunos de los factores que afectan la colocación de soluciones mecánicas son la presencia de sólidos en el pozo, el ángulo de desviación, la existencia de flujo cruzado al interior del yacimiento y las condiciones de temperatura del subsuelo (2019, p. 7). También exponen que las soluciones mecánicas expuestas anteriormente se aplican dependiendo el tipo de problema que se tenga al interior del pozo, por ejemplo, si se tiene una alta producción de $\mathrm{H}_{2} \mathrm{~S}$, que causa corrosión sobre las tuberías (Hausler, R. H. 2004), una posible solución es la aplicación de cemento justo en la zona donde se sabe existe la fuga de este gas (p. 9). Para finalizar, los autores enuncian que la producción excesiva de agua causa numerosos problemas económicos para las compañías productoras y que por lo tanto es importante distinguir el agua que es necesaria del agua indeseada y su punto de entrada al pozo, para, basándose en esta información, seleccionar la solución adecuada ya sea química o mecánica (p. 9-10).

\section{Solución química}

Baily B. et al. sostienen que este tipo de tratamiento requiere de una gran precisión a la hora del posicionamiento; dicen además que el uso de equipos de Coiled Tubing apoyado con empaques inflables o con doble inyección, ayuda a evitar que el fluido de tratamiento se extienda a zonas donde no se desea que se posicione. En su revisión enuncian que existen varios tipos de químicos que pueden ayudar en el control de la producción de agua como cementos, geles rígidos, polímeros entrecruzados, geles, entre otros que pueden ser inyectados usando la tubería de producción vía Bullheading, con el propósito de ahorrar algunos costos durante el proceso (2000, p. 16).

van der Hoek J.E., Botermans W. y Zitha. L.J., enuncian que poner sistemas gelificantes totalmente sellantes en los estratos que producen agua es una posible solución al problema económico y técnico que acompaña la excesiva producción de agua. (2001, p. 1).

Botermans C.W., D. W. van Batenburg y J. Bruining anuncian que existen sistemas, llamados Modificadores de Perfil (RPM por sus siglas en ingles), los cuales reducen la permeabilidad relativa al agua sin afectar significativamente la permeabilidad relativa al aceite (2001, p. 1). 
Syndansk R.D. y Seright R.S. afirman que la capacidad de la acrilamida para reducir la permeabilidad relativa del agua más que la permeabilidad relativa del aceite fue reconocida desde el año 1964, por Sandi Ford. Luego afirman que el mecanismo por el cual esto sucede fue el centro de muchas investigaciones y proceden a explicar que la razón por la cual este tipo de soluciones ha resultado tan atractiva para la industria de los hidrocarburos es que (al menos en el caso de los tratamientos de reducción desproporcionada de la permeabilidad -DPR por sus siglas en inglés) pueden ser inyectados sin la ayuda de equipo adicional, lo que resulta en ahorros de tiempo, equipo y personal además del hecho de que cuando se tienen completamientos especiales, como paquetes de grava, una inyección diferente al BullHeading es muy difícil de conseguir (2006, p. 2).

Lei G., Li L. y Nasr-El-Din H.A. enuncian que históricamente se han usado geles inorgánicos, geles no poliméricos, geles silicatados, geles a base de polímeros entrecruzados, geles de poliacrilamida y Cromo, Geles de dispersión Coloidal, microgeles y geles particulados para controlar patrones de inyección, disminuir el corte de agua, aumentar el tiempo de ruptura, reparar fugas en el casing, reducir la permeabilidad al agua $\mathrm{y} / \mathrm{o}$ incrementar la eficiencia de barrido (2010, p. 1-2).

Manrique E. et al. enuncian que los químicos inorgánicos son usados para sellar perdidas de circulación, dirigir la dirección del fluido durante una cementación forzada y ayudar en la consolidación de formaciones débiles, ya que estos pueden ser inyectados más fácilmente, no son caros y tienen una mayor profundidad de inyección dentro del yacimiento. (2014, p. 3).

Xindi S. y Bai B. citan varios mecanismos mediante los cuales se puede alcanzar el efecto de reducir la producción de agua en pozos horizontales, encontrándose entre ellos, los polímeros sellantes que consisten en partículas de gel preformadas las cuales absorben hasta 400 veces su peso en agua (Peirce J. W. et al., 2014). Otras soluciones descubiertas por estos investigadores en su propia revisión bibliográfica incluyen geles entrecruzados de poliacrilamida y cromo, micro cemento (el cual tiene tamaño de partícula menor a 10 micrómetros), agente sellante HWSO (compuesto de acrilamida, akilbromil y un copolímero de Etilenmetacrilato) (2017, p. 3).

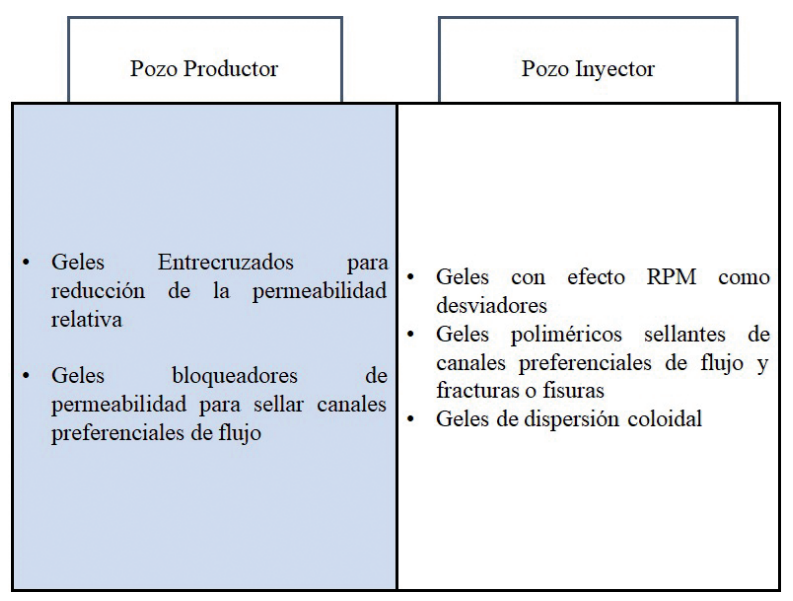

Figura 3. Tipos de geles a usar para control de agua en pozos inyectores o productores.

\section{Geles}

En este apartado se plantea una revisión en orden cronológico de las conclusiones a las que han llegado diversos investigadores en cuanto a los tipos de geles para control de agua que existen, como están formados y que usos tienen al ser inyectados al yacimiento.

Sparlin D.D. explica que los agentes entrecruzantes son sales multivalentes y que se ha descubierto que los polímeros aniónicos son más reactivos que los polímeros menos iónicos. Debido a que un polímero aniónico reacciona muy rápido con el agente entrecruzador ambos compuestos deben ser añadidos por separado situación contraria en el caso de los polímeros no iónicos (1976, p. 5), lo que genera algunos beneficios logísticos y económicos al momento de realizar la inyección al yacimiento.

Syndansk R.D. explica cómo son inyectados los tratamientos de geles entrecruzados en un yacimiento con problemas de producción de agua. Los geles son un único sistema de fluidos en superficie, el cual es formado simplemente por la adición de un agente entrecruzador a un polímero en una solución acuosa. Estos compuestos reaccionan algún tiempo después de ser mezclados para formar una red tridimensional conocida como gel, que es estable por largos periodos de tiempo en amplios rangos de temperatura. (1988, p. 1). 


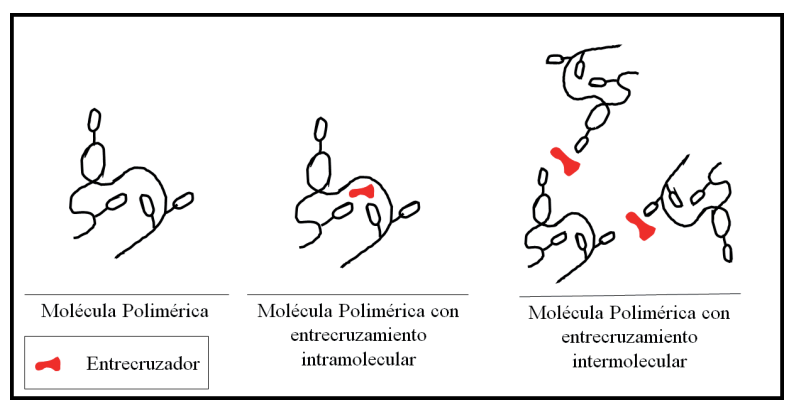

Figura 4. Explicación grafica de las reacciones de entrecruzamiento. (Modificado de Spildo K., Skauge A., Skauge T., 2010).

Borling D., Chan K., Hughes T. y Syndansk R.S. recuentan que desde los años 70 se han hecho investigaciones sobre los sistemas gelificantes y en algunos de ellos se determinó que originalmente son una mezcla fluida de dos componentes: un polímero de alto peso molecular y un entrecruzador. Lo que sucede es que cada molécula de entrecruzador se une con dos o más moléculas del polímero uniéndolas químicamente entre sí, resultando en una red tridimensional de moléculas poliméricas interconectadas; debido a esto la mezcla deja de comportarse como un fluido para pasar a ser un gel. (1994, p. 3).

Whittington L.E., D.G. Naae y R.W. Braun enuncian que factores como el $\mathrm{pH}$, la temperatura, la composición del agua de preparación, la tasa de inyección, y la composición química deben ser consideradas en el diseño del sistema gelificante porque podrían afectar su reactividad y efectividad para controlar la producción de agua (1994, p. 2).

Kakadjan S., Rauseo O. y Mejias F. enuncian que los polímeros entrecruzables atraviesan varias fases durante su transición de ser un líquido a un gel, las cuales se diferencian por su grado de consistencia (1999, p. 1).

McCall S., Li X. y Willhite recalcan que los geles poliméricos son aplicados a pozos de inyección y de producción para modificar el flujo de los fluidos del yacimiento, para reducir la producción de agua y mejorar la producción de aceite (2007, p. 1).

Romero L., Mánalo F. y Cantas A. recuentan que los geles altamente entrecruzados son usados en yacimientos con alta permeabilidad para controlar la pérdida de fluido durante el completamiento y las operaciones de workover. Enuncian además que los geles entrecruzados también son usados para detener la alta producción de gas o agua desde los pozos de producción y para controlar el patrón de flujo en la cercanía de los pozos inyectores; Explican que los geles son redes poliméricas que tienen las propiedades cohesivas de los sólidos y las propiedades difusivas de los líquidos; La tasa a la cual se forma el gel determina que tan lejos del pozo puede colocarse el tratamiento, por tanto se convierte en un parámetro a controlar. Finalmente, los autores enuncian que la consistencia del gel se relaciona con la máxima caída de presión que el gel puede soportar dentro del medio poroso (2008, p. 1). El tiempo que tarda la solución gelificante para iniciar la transición entre el estado líquido a un estado rígido (con alto entrecruzamiento) es conocido como tiempo de gelificación (Salgaonkar L. y Das., 2012, p. 2).

Vásquez J. y Eoff L. enuncian que los fluidos de RPM fueron introducidos a la industria de los hidrocarburos como una opción para reducir selectivamente la producción de agua; este tipo de tratamiento se hizo popular para las empresas operadoras por su simplicidad y porque no requieren de ningún tipo de aislamiento durante su aplicación. (2013, p. 1).

Das P., Patil P. y Agashe S. afirman que cuando se desea inyectar un tratamiento que selle totalmente el flujo en caminos de alta permeabilidad, se debe inyectar antes un desviador para que evite que el tratamiento sellante se posicione en zonas que se desea mantener en producción, el cual podría ser un RPM según los investigadores (2013, p. 1).

Manrique E., et al. (2014, p. 2-3) hacen una revisión de los tipos de geles disponibles:

1. Geles poliméricos: moléculas poliméricas se unen entre sí a través de entrecruzadores formando un gel viscoso dentro del yacimiento. Los geles poliméricos pueden usarse para con fines de sellado o de DPR

2. Geles monoméricos: son monómeros de acrilato que se polimerizan dentro del yacimiento y pueden o no ser entrecruzados; el problema principal con estos geles es su costo y que no son amigables con el medio ambiente.

3. Geles silicatados: tienen una alta penetración dentro del yacimiento ya que tienen una baja viscosidad y una buena estabilidad química y térmica, además de su bajo costo y que son amigables con el medio ambiente.

4. Geles particulados preformados: son usados en aplicaciones donde se requiere tratar fracturas, 
canales y son buenos para soportar altas temperaturas, salinidades y variadas condiciones de $\mathrm{pH}$.

5. Geles coloidales: son geles que requieren bajas concentraciones de polímero y entrecruzador, lo cual hace que los volúmenes de tratamiento sean más económicos de inyectar. Su aplicación en campo está limitada a bajas temperaturas de yacimiento y baja salinidad del agua de formación.

El-Karsani S.M., Al-Almuntasheri G.A., Sultan A.S. y Hussein H.A. enuncian que los geles poliméricos son uno de los métodos más usados en los yacimientos de petróleo para superar el problema del exceso en la producción de agua. Los geles poliméricos están basados en una mezcla de agua, polímero y un entrecruzador la cual es inyectada a la zona objetivo a través de muchas técnicas como el Bullheading o el Coiled Tubing (Jaramillo O.J., Romero R., Lucuara G., Ortega A., Milne A. y Lastre M., 2010). Cuando la mezcla se inyecta ocurre una reacción de entrecruzamiento que une el polímero y el entrecruzador, la cual se ve afectada por la temperatura, la concentración de los componentes, el tiempo, entre otros factores (2015).

Bai B., Zhou B. y Yin M. dividen los tratamientos con geles poliméricos en dos: bloqueadores de permeabilidad, los cuales son usados cerca a la cara del pozo (Simjoo M., Koohi A.D., Vafaie-Sefti M. y Zitha P.L.J., 2009) y los modificadores de permeabilidad relativa que tienen un mayor alcance dentro de la formación $(2015$, p. 1).

Aldhaheri M.N., Wie M., Bai B. y Alsaba M. (2016, p. 5), toman los geles poliméricos y los dividen en tres grandes tipos:

1. Geles: son formados usando poliacrilamidas de alto peso molecular y un entrecruzador.

2. Geles de dispersión coloidal: son formados por bajas concentraciones de poliacrilamida hidrolizada entrecruzada con citrato de aluminio o citrato de cromo. El hecho de usar bajas concentraciones de estos compuestos forma geles débiles los cuales no alcanzan a formar una red si no burbujas de gel que son casi esféricas y pueden fluir bajo un amplio rango de presiones diferenciales.

3. Geles débiles: son geles menores concentraciones de polímero y entrecruzador lo que hace que el gel sea lo suficientemente móvil para ser inyectado más allá de la cara del pozo.
San Y. et al. comparan la estabilidad térmica que le confiere al gel el hecho de tener un entrecruzador metálico o uno orgánico, concluyendo que los polímeros con entrecruzadores metálicos no tienen tanta resistencia térmica y que además los tiempos de gelificación tienden a ser mayores que cuando se usan entrecruzadores orgánicos (2017, p. 1).

Zhu D., Bai B. y Hou J. (2017), dividen los geles en tres tipos así:

1. Geles poliméricos entrecruzados en yacimiento: es una solución muy convencional y consiste en el mezclado de un solvente, un polímero $\mathrm{y}$ un entrecruzador (metálico u orgánico) que reaccionan químicamente, en un periodo posterior al mezclado, conocido como tiempo de gelificación, para formar una estructura tridimensional. Se distinguen los siguientes tipos: geles de poliacrilamida, geles poliméricos sintéticos y geles poliméricos naturales, como los biopolímeros (p. 1-2).

2. Geles preformados, entre los cuales se encuentran los geles preformados, geles particulados preformados, microgeles y sistemas dispersos. Su ventaja sobre los geles poliméricos entrecruzados es que no existe la necesidad de controlar el tiempo de gelificación de la mezcla ya que el proceso de gelificación sucede antes de la inyección (p. 15).

3. Geles espumosos: pueden ser usados para taponar los caminos de alta permeabilidad durante una inyección de agua. Estos geles son creados de manera similar a una espuma acuosa. Su ventaja sobre los sistemas convencionales consiste en que se requieren menores cantidades del gelificante, lo cual reduce costos (p. 19).

\section{Geles de DPR}

Dentro de la revisión realizada, la primera vez que se menciona el uso de químicos que causan una reducción desproporcionada en la permeabilidad relativa al agua, sucede en 1997, cuando Liang J.T. y Seright R.S., enuncian que existen algunas explicaciones que pueden ayudar a entender por qué los agentes de DPR tienen este efecto (p. 2). Posteriormente Nilson S., Stavland A., y Jonsbraten H.C., en 1998, concluyeron basados en sus experimentos, que el efecto de DPR es causado porque existen caminos diferenciados por los cuales fluyen el petróleo y el agua (p. 1), lo cual podía ser evidenciado al variar la mojabilidad de la muestra evaluada y la fase del solvente en el cual se agregan el polímero y el entrecruzador (p. 6). En 1999 Zaitoun A., 
Kohler N., Bossie-Codreanu y Denys K., enuncian que a pesar de que en el momento de su publicación existía alguna controversia sobre la explicación de cómo los RPM funcionan a nivel del poro, existían dos escuelas de pensamiento principales: una que se apoyaba en la proporción de fluido en la mezcla (fase del solvente) y otra que se apoyaba en los efectos de pared de roca sobre la solución gelificante (p. 2).

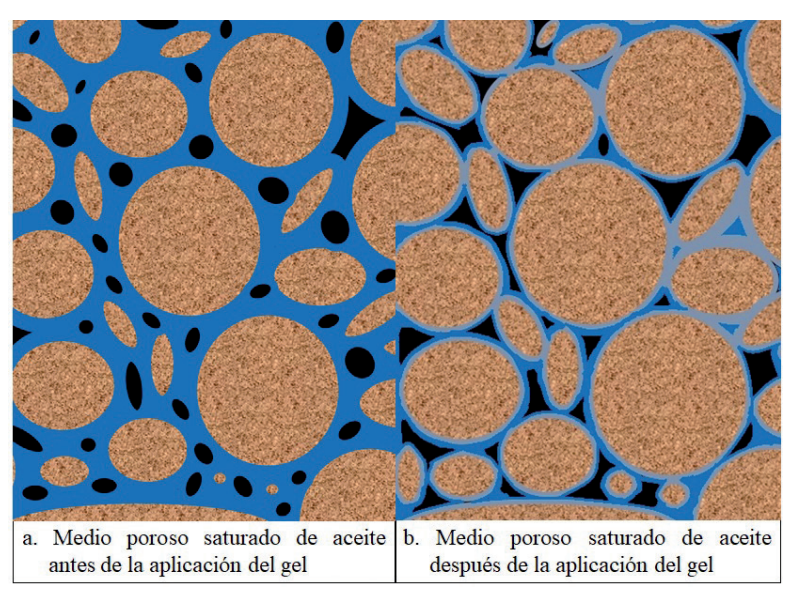

Figura 5. Ilustración de los efectos de la pared de roca sobre el tratamiento gelificante. (Modificado de Liang J.T. y Seright R.S. 1997).

Syndansk R.D. y Seright R.S. hacen una equivalencia entre el termino RPM (Relative Permeability Modifiers) y el termino DPR (Disproportionate Permeability Reduction), cuando enuncian que: "RPM también es referido como Reducción desproporcionada de la permeabilidad (DPR)" (2006, p. 1), pero hacen la salvedad al enunciar que algunos investigadores se refieren a los agentes de DPR al hablar de los geles fuertes, mientras que guardan el termino RPM para soluciones de geles débiles o polímeros que causan una sutil reducción en la permeabilidad relativa al agua. (2006, p. 1). Vásquez J. y Eoff L. enuncian que la mejor forma de aplicar una reducción en la permeabilidad relativa al agua más que al aceite es posicionar el gel en un estrato que esté completamente saturado con agua ya que inyectarlo en un estrato que tenga una alta saturación de aceite no tendrá ningún efecto en la producción de agua. (2013, p. 3).

\section{Parámetros que afectan el entrecruzamiento}

En esta sección se desea explorar la manera que tuvieron los diferentes autores consultados de enunciar los factores que afectan el proceso de gelificación, en el cual el polímero y el entrecruzador reaccionan para formar una red tridimensional rígida que conocemos como gel.

Sparlin D.D., enuncia que la litología tiene algún efecto sobre la afinidad entre el polímero y la matriz de roca, observando que los sitios aniónicos de la poliacrilamida estaban atraídos por la matriz de los carbonatos, resultando en una mayor cantidad de polímero retenido en la matriz de carbonato que en las de arenisca; las arcillas por su parte también generaron alguna respuesta en la retención del polímero, debido a que el mineral contenido en este tipo de roca absorbe demasiada agua, por lo cual el polímero se quedaba adherido a los granos de la roca $(1976$, p. 4). El autor observó también que el pH tiene alguna influencia sobre la viscosidad de la solución, observándose que para $\mathrm{pH}$ alcalinos (7-14) la solución tiende a ser más viscosa y reaccionar con mayor velocidad (p. 5). Otro factor que afecta la velocidad de entrecruzamiento es el número de sitios reactivos en el polímero, el cual se ve reflejado en su carga iónica, obteniéndose que para polímeros no iónicos la velocidad de reacción es más baja (p. 5). Borling D., Chan K., Hughes T. y Syndansk R.S. (1994) aportan que el aumento en la temperatura del sistema aumenta la velocidad de reacción, por lo que, a temperaturas muy elevadas la gelificación puede hacerse difícil de controlar (Araujo Y. C., y Araujo M., 2018).

Syndansk R.D. (1988) encontró que la presencia de hidrocarburos, la litología del yacimiento (areniscas y carbonatos) los materiales propios de la operación, como las tuberías y los tanques de almacenamiento, no generaban un efecto adverso en el proceso de entrecruzamiento o en la consistencia del gel. El $\mathrm{H}_{2} \mathrm{~S}$ fue para el autor una sorpresa ya que, por su reactividad, se esperaba que retrasara el proceso de gelificación, lo cual no ocurrió en sus experimentos; por otro lado, en el estudio realizado por el autor, la presencia de sal en el agua de preparación si tuvo algún efecto sobre el proceso de entrecruzamiento y la consistencia final del gel.

Al Muntasheri G.A., Nasr El-Din H.A., Peters J.A. y Zitha. L.J., hacen primero una claridad en cuanto a la clasificación de los geles que pueden obtenerse en función del origen del entrecruzador, en orgánicos y metálicos. Enfocándose y continuando el desarrollo de la teoría de su artículo con los entrecruzadores orgánicos; identifican que el mecanismo de reacción entre el polímero y el entrecruzador es la formación de un enlace covalente que los une y que la hidrólisis 
del entrecruzador puede ser el paso previo al proceso de entrecruzamiento (en el caso de usar polietileimina como entrecruzador) (2003, p. 5).

Brattekas B. et al. enuncian que los fluidos del yacimiento pueden interferir en el proceso de gelificación, mientras que los geles preformados no tienen ningún tipo de actividad frente a las condiciones fisicoquímicas de los yacimientos (2003, p. 1).

Bai B., Zhou B. y Yin M, mencionan que el grado de hidrolisis es la proporción de grupos amida que se transforman en carboxilos los cuales reaccionan con el entrecruzador (en el caso de usar Poliacrilamida Hidrolizada -HPAM por sus siglas en inglés- como polímero), es decir, el paso previo al entrecruzamiento es la hidrólisis $(2015$, p. 2). Luego enuncian que en un principio (cerca a los años 1970) se utilizaba el sulfato de aluminio con entrecruzador para la poliacrilamida parcialmente hidrolizada, pero que el entrecruzador el sulfato de aluminio- era demasiado dependiente del $\mathrm{pH}$ observándose que en ambientes ácidos no había reacción entre ambos compuestos mientras que cuando la mezcla de compuestos era inyectada al yacimiento el $\mathrm{pH}$ tendía a aumentar rápidamente por lo que el entrecruzamiento se producía sin ningún control. Por esta razón, a partir de estos descubrimientos se cambió el entrecruzador a el Cromo, el cual mostraba tiempos de gelificación más retardados (aunque variables con la temperatura), como consecuencia de este cambio la solución gelificante podía ser inyectada más profundo dentro del yacimiento $(2015$, p. 2$)$.

Sun Y. et al. identifican tres periodos durante el proceso de entrecruzamiento o gelificación $(2017$, p. 4):

- Primer periodo o periodo de inducción: en el cual no se observan reacciones de entrecruzamiento, probablemente porque los compuestos están preparándose químicamente para reaccionar.

- Segundo periodo o periodo de entrecruzamiento rápido: es el momento en cual se producen la mayor parte de las reacciones de gelificación.

- Tercer periodo, o periodo estable: en el cual se detienen las reacciones de entrecruzamiento por falta de reactivos o porque todos los sitios donde se puede realizar el enlace químico entre compuestos están ocupados; en este periodo se alcanza el máximo grado de consistencia que puede tener el gel.

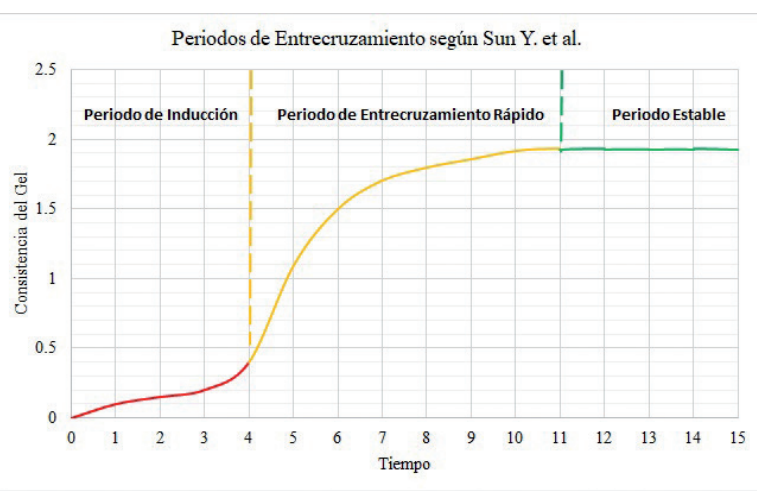

Figura 6. Tres periodos del entrecruzamiento (Modificado de Sun Y. et al.)

Los autores identifican que hay varias condiciones que afectan no solo la velocidad de entrecruzamiento sino también la consistencia que alcanzan los geles, entre ellos el pH de la solución, la salinidad del agua de preparación, las concentraciones de reactivo y la temperatura $(2017$, p. 10$)$.

Zhu D., Bai B. y Hou J. indican que el tiempo de gelificación debe ser acomodado para realizar las operaciones de inyección y posicionamiento del gel y los factores que pueden influenciar esta respuesta son la temperatura, la salinidad, el $\mathrm{pH}$ y la concentración de los reactivos $(2017$, p. 3$)$.

Unomah M. et al. se concentran en lo que sucede después del entrecruzamiento, la sinéresis, que es un proceso en el cual el gel expulsa agua y se encoje al mismo tiempo, el encogimiento hace que el gel deje de ser efectivo para los propósitos del control de agua. La sinéresis es causada por la interacción entre el gel y los iones divalentes del agua de formación como el Calcio y el Magnesio (2018, p. 3).

\section{Parámetros que influyen en la efectividad del tratamiento}

El propósito de este capítulo es revisar cuales son los parámetros más relevantes que influyen en la efectividad de un tratamiento para control de la excesiva producción de agua usando modificadores de permeabilidad relativa o agentes de reducción desproporcionada en la permeabilidad, según lo identificado por diferentes autores.

Vásquez J. y Eoff L., enuncian que el candidato ideal para la inyección de tratamiento con efectos de RPM son yacimientos estratificados que no estén comunicados entre sí (2013, p. 4). También enuncian 
que estratos en los cuales la saturación de hidrocarburos es muy alta no son buenos candidatos para este tipo de tratamientos, ya que el fluido inyectado no tendrá ningún efecto en el perfil de producción; además de esto los estratos en los cuales la saturación de agua móvil es muy alta tampoco son buenos candidatos debido a que cuando el agua llegue a la cara del pozo productor disminuye la permeabilidad relativa al aceite lo cual disminuye su tasa de flujo y hace del tratamiento un fracaso; otro caso de fracaso es cuando se tiene flujo cruzado entre los estratos, el problema radica en que existirá un momento después de haber de inyectado el tratamiento en el cual el agua dejará de moverse al interior del estrato productor buscando el pozo productor (a causa del tratamiento inyectado) y empezará a migrar hacia estratos que le ofrezcan una menor resistencia logrando incorporarse de nuevo a los fluidos producidos del yacimiento (p. 4).

Zaitoun A. et al. (1999) dicen que los contrastes de permeabilidad favorecen la colocación del tratamiento en las zonas que producen mayor cantidad de agua por ser las que tienen mayor Permeabilidad relativa, pero estos estratos de diferente permeabilidad deben tener algún tipo de barrera entre sí, para evitar que el agua que perdió movilidad por efectos del tratamiento migre a zonas donde el tratamiento no pudo ser colocado.

Además del yacimiento donde se desea realizar la inyección, debe tenerse en cuenta el sistema químico a inyectar (Sampaio M.A., Montoya S.D. y González J.C., 2018); Lei G., Li L. y Nasr-El-Din H.A. enuncian que un sistema químico ideal para controlar la producción de agua debe ser tolerante a las altas salinidades y la alta dureza del agua de formación, debe ser compatible con cualquier tipo de agua, debe tener estabilidad térmica, baja viscosidad y la capacidad para penetrar en el espacio poroso $(2010$, p. 2$)$.

Hatzignatiou D.G., Helleren J. y Stavland A. dan prioridad al diseño del gel para obtener una mayor efectividad en la etapa de tratamiento; entre los factores que identifican como importantes se encuentran la consistencia, el tamaño de las partículas del gel y el tiempo de gelificación (2014, p. 3).

Otro parámetro de importancia a considerar es el proceso mismo de inyección; este proceso hace que la solución gelificante se degrade mecánicamente. Sparlin D.D. encontró que a pesar de que los reactivos se degraden, una vez entrecruzados, su capacidad para reducir la producción de agua no se ve alterada, por lo que la tasa de inyección del tratamiento puede ser tan alta como se desee, obteniendo la ventaja de inyectar el tratamiento más profundo en la formación (1976, p. 7). El tamaño de los poros o fracturas en los cuales se inyecta la solución gelificante también afecta la efectividad del tratamiento, al igual que la saturación de fluidos del medio, las partículas sólidas ajenas a la formación, la suciedad y los restos de la perforación pueden crear un efecto de daño haciendo que el tratamiento se posicione en zonas no deseadas (Sparlin D.D., 1976, p. 8). Stavland A., Andersen L.I., Standoy B., Tjomsland T. y Metrabu A.A., (2006, p. 5) encontraron que las propiedades de inyectividad del polímero, la diferencia entre la viscosidad del tratamiento y los fluidos del yacimiento, la permeabilidad y el tamaño de los poros también afecta la colocación del tratamiento. El factor principal que caracteriza un gel para control de la producción de agua es la profundidad horizontal que se alcanza durante la inyección, ya que este factor determina la capacidad que tendrá el gel de soportar las caídas de presión que se generan en la fase de producción (van der Hoek J.E., Botermans W. y Zitha. L.J., 2001, p. 1); otro factor de importancia es la consistencia que alcanza el gel, (la cual viene determinada por la cantidad de entrecruzador, compuesto que determina el número de puntos de unión con el polímero); como regla general, un gel más rígido es deseable para detener el agua que fluye en el espacio poroso (van der Hoek et al., 2001, p. 6).

Como había sido ilustrado en la sección precedente, las arcillas hacen que el solvente en el cual se diluyen el polímero y el entrecruzador se adhiera a la roca (Sparlin D.D., 1976, p. 4), por lo que se termina con volúmenes menores de tratamiento y una concentración más elevada de reactivos, lo que podría conducir a geles más rígidos que reduzcan en mayor proporción la producción de fluidos de la zona (Sparlin D.D., 1976, p. 7), incluyendo entre estos fluidos al petróleo.

En la escala del poro, la mojabilidad de la roca, el tamaño de los poros y la fracción del espacio poroso ocupada por el gel tienen alguna incidencia en la efectividad del tratamiento (Nilsson S., Stavland A., y Jonsbraten H.C., 1998, p. 12).

Debido a que en algunos casos un factor a evitarse es el taponamiento de los poros, debe seleccionarse adecuadamente el peso molecular y el tamaño de las moléculas del polímero (Seright R.S., Fan T., Wavrik K. y de Carvalho Balaban R., 2010, p. 6); por lo general se usan poliacrilamidas parcialmente hidrolizadas con pesos moleculares entre 6 y 20 millones de Daltons (Niño L., de Sousa F. V. H. y Rosangela M., 2019). 
Awad Q., Ibrahim A., Salah R. y Bakheet A. enuncian que la temperatura, las condiciones de inyección y la penetración del gel en el espacio poroso son determinantes en su efectividad; los autores además afirman que hay más factores que intervienen en la penetración del tratamiento en el espacio poroso, los cuales son la presión del estrato, su porosidad, permeabilidad y la saturación irreducible de fluidos en la profundidad de inyección (2010, p. 3).

Finalmente, Zaitoun A. et al enuncian que para mantener los mismos niveles de producción que se tuviera antes de haber inyectado el tratamiento, las caídas de presión deben ser ajustadas y que debido a que el gel debe soportar las condiciones del yacimiento se le debe diseñar para ser estable a esa temperatura y a las nuevas condiciones de presión diferencial entre el yacimiento y el pozo (1999, p. 2-3).

\section{Conclusiones}

1. La producción excesiva de agua es un problema que afecta a las compañías operadoras de manera negativa, ya que incrementa los costos de producción del petróleo, disminuye la vida productiva de pozos los productores y aumenta la capacidad necesaria para realizar el tratamiento de los fluidos del yacimiento.

2. Para implementar de manera correcta una solución al problema de producción de agua se debe conocer muy bien la situación problema a través de la adquisición de la mayor cantidad posible de información, la cual se encuentra en registros de producción, registros de pozo, curvas de declinación, análisis nodal, gráficos tipo, entre otros que contienen información del pozo y su producción de fluidos.

3. Entre las soluciones a los problemas de control de agua se encuentran las soluciones mecánicas, que causan una modificación en el pozo que ayuda a reducir la cantidad de agua que ingresa desde el yacimiento; también se encuentran las soluciones químicas como los modificadores de perfil, las soluciones gelificantes, los químicos inorgánicos, que se sitúan en la formación o en la cara del pozo y ayudan a controlar la cantidad de agua producida.

4. Se puede concluir que los geles de DPR son una opción viable para reducir la permeabilidad relativa al agua de zonas en las cuales se desea aumentar la producción de petróleo y que el hecho de que no se requiera equipo adicional y que exista una amplia literatura la hacen una técnica atractiva para las compañías operadoras que buscan reducir los costos asociados a la producción y tratamiento de agua.

5. Los parámetros que pueden afectar la efectividad de un tratamiento con geles entrecruzables son la litología del yacimiento, la temperatura, la salinidad del agua de formación y la composición de los compuestos químicos usados en la formulación del gel.

\section{Siglas}

ICD: Inflow Control Device

WOR: Water Oil Ratio

WOC: Water Oil Contact

RPM: Relative Permeability Modifiers

DPR: Disproportionate Permeability Reduction

HPAM: Partially Hydrolyzed Polyacrylamide

\section{Referencias}

Al Muntasheri G.A., Nasr El-Din H.A., Peters J.A. y Zitha. L.J. (2003). Investigation of a HighTemperature Organic Water-Shutoff Gel: Reaction Mechanisms. SPE Journal. 1-8.

AldhaheriM.N., WeiM.yBaiB.(2016). Comprehensive guidelines for the application of in situ polymer gels for injection well conformance improvement based on field projects. SPE improved oil recovery conference held in Tulsa, Oklahoma. 1-27.

Aldhaheri M.N., Wie M., Bai B. y Alsaba M. (2016). A Roadmap to Successfully Select a Proper Gel Treatment Technology. SPE Kingdom of Saudi Arabia Technical Symposium and Exhibition, held in Dammam, Saudi Arabia. 1-30.

Aldhaheri M.N., Wie M., Bai B. y Alsaba M. (2016). An Advanced Selection Tool for the Application of In-Situ Polymer Gels for Undiagnosed Injection Wells. SPE Kingdom of Saudi Arabia Technical Symposium and Exhibition, held in Dammam, Saudi Arabia. 1-25.

Alzate D., León. J. M., Cabrera F. y Manrique E. (2017). Evidencias de la formación de CDG y posibles interpretaciones de pruebas de desplazamiento en muestras de núcleos. Revista Fuentes: El Reventón Energético, volumen 15 ( $\left.\mathrm{n}^{\circ} 2\right)$. 31-47

Araujo Y. C., y Araujo M. (2018). Polymers for application in high temperature and salinity reservoirs - critical review of properties and 
aspects to consider for laboratory screening. Fuentes: el Reventón Energético, volumen $16\left(\mathrm{n}^{\circ}\right.$ 2). 55-71.

ARPEL, (s.f.). disposición y tratamiento del agua producida. $1-120$

Awad Q., Ibrahim A., Salah R. y Bakheet A. (2010). Risk Analysis and Decision Making in Relative Permeability Modifier WaterShut-off Treatment. North Africa Technical Conference and Exhibition held in EL Cairo, Egypt. 1-14.

Bai B., Zhou B. y Yin M. (2015). A comprehensive review of polyacrylamide polymer gels for conformance control. Petroleum exploration and development. 1-8.

Baily B., Crabtree M., Tyrie J., Elphick J., Kuchuk F., Romano C. y Roodhart L. (2000). Water control. Oilfield Review. 1-22.

Borling D., Chan K., Hughes T. y Syndansk R.S. (1994). Pushing out the oil with conformance control. Oilfield review. 1-15.

Botermans C.W., D. W. van Batenburg y J. Bruining. (2001). Relative permeability modifiers: Myth or reality? Presented at the SPE European Formation Damage Conference held in the Hage, The Netherlands. 1-13.

Brattekas B., Pedersen S.G., Nistov H.T., Haugen A., Graue A., Liang J.T. y Seright R.S. (2015). Washout of Cr (III)-Acetate-HPAM Gels from Fractures: Effect of Gel State During Placement. SPE improved Oil recovery Symposium held in Tulsa, Oklahoma. 1-11.

Busaidi K. y Bhaskaran H. (2003). High-Water Cut: Experience and Assessment in PDO. SPE Annual Technical Conference and Exhibition held in Denver, Colorado, USA. 1 - 7

Castro W.J. y Ordoñez D.F. (2019). Evaluación técnicofinanciera de los efectos de los modificadores de permeabilidades relativas en el campo la Cira Infantas. Fundación Universidad de América, Facultad de Ingenierías, Programa de Ingeniería de Petróleos. 1-166.

Daneshy A.A., Krasnov V.A. y Zimin S.V. (2010). ICD Design: revising objectives and techniques SPE
Asia Pacific Oil and Gas Conference and exhibition held in Brisbane, Queensland, Australia. 1-11.

Das., Patil. y Agashe S. (2013). Application of relative permeability modifier for sealant diversion. The Noth Africa technical conference and Exhibition held in El Cairo, Egypt. 1-10.

El-Karsani K.S.M., Al-Muntasheri G.A. y Hussein I.A. (2014). Polymer Systems for Water Shutoff and Profile Modification: A Review Over the Last Decade. 1-15.

El-Karsani S.M., Al-Almuntasheri G.A., Sultan A.S. y Hussein H.A. (2015). Gelation of a Water-Shutoff Gel at High Pressure and High Temperature: Rheological Investigation. SPE Journal. 1-10.

Eoff L., Dalrymple D., Everett D. y Vasquez J. (2006). Worldwide field applications of a polymeric gel system for conformance applications. SPE International symposium and exhibition on Formation damage control, Lafayette, Louisiana. $1-5$.

Erazo-Bone R., Escobar-Segovia K., España F. y Hernández T. (2019). Completaciones inteligentes en pozos inyectores de agua utilizando ICD para reducir el efecto de interdigitación viscosa. Revista Fuentes: El Reventón Energético, volumen 17 (n 2). 51-61.

Garzón M. F., Barrero L. P., Portilla H. E., Castillo G. (2014). Efecto del completamiento con dispositivos de control de influjo sobre la producción de un pozo horizontal de un campo colombiano. Fuentes: el Reventón Energético, volumen $12\left(n^{\circ} 1\right)$. 33-47.

Green D. W. y Willhite G. P. (1998). Enhanced Oil Recovery. Society of Petroleum Engineers. Richardson Texas. $1-561$.

Hatzignatiou D.G., Helleren J. y Stavland A. (2014). Numerical Evaluation of Dynamic Core Scale Experiments of Silicate Gels for Fluid Diversion and Flow Zone Isolation. SPE production and operations. 1-17.

Hausler, R.H.(2004). Contribution to the Understanding of H2S Corrosion. NACE International. 1-25. 
Jaramillo O.J., Romero R., Lucuara G., Ortega A., Milne A. y Lastre M. (2010). Combining stimulation and water control in high-watercut wells. SPE International Symposium and Exhibition on Formation Damage Control held in Lafayette, Louisiana, USA. 1 - 10.

K.S. Chan (1995). Water Control Diagnostic plots. SPE annual technical conference and exhibition held in Dallas, U.S.A. 1-9.

Kakadjan S. y Rauseo O., Mejias F. (1999). Dynamic Rheology as a Method for Quantify Gel Strength of Water Shutoff Systems. SPE international symposium on oilfield chemistry held in Houston, Texas. 1-11.

Lei G., Li L. y Nasr-El-Din H.A. (2010). New Gel Aggregates for Water Shut-off Treatments. SPE Improved Oil Recovery Symposium held in Tulsa, Oklahoma. 1-15.

Liang J.T. y Seright R.S. (1997). Further Investigations of Why Gels reduce Water permeability more than oil permeability. SPE Production and Facilities. 1-6.

Manrique E., Reyes S., Romero J., Aye N., Kiani M., North W., Thomas C., Izadi M., Roostapour A. y Muniz G. (2014). Colloidal Dispersion Gels (CDG): Field Projects Review. SPE EOR conference at Oil and Gas West Asia held in Muscat, Oman. 1-13.

McCool S., Li X. y Willhite. (2007). Effect of shear on flow properties during placement and on syneresis after placement of a polyacrylamide-chromium acetate gellant. SPE international Symposium on oilfield chemistry held in Houston, Texas. 1-16.

Mejia H. D. (2016). Estudio técnico y económico de la inyección de químicos como método para reducir la relación Agua-Petróleo en yacimientos con empuje hidráulico. Universidad Industrial de Santander. Facultad de ingenierías FísicoQuímicas. Escuela de Ingeniería de Petróleos. Especialización en Producción de Hidrocarburos. $1-99$.

Mendoza J.R. (2013). Diseño y evaluación de modificadores de permeabilidad relativa como alternativa para el control de agua. Universidad Nacional de ingeniería, Facultad de Ingeniería de Petróleo, gas natural y petroquímica. 1-134.
Misha A., Abbas S., Braden J., Hazen M., Li G., Peirce J. y Smith D.D. (2016). Comprehensive review of fracture control for conformance improvement in the Kuparuk River Unit -Alaska. SPE improved oil recovery conference held in Tulsa, Oklahoma, USA. 1-24

Montes A., Carreño W. y Guio M. (2016). Aspectos de la perforación de pozos complejos en piedemonte en tiempos de crisis. Fuentes: El Reventón Energético, volumen 16 ( $\left.\mathrm{n}^{\circ} 1\right) .87-97$.

Nilsson S., Stavland A., y Jonsbraten H.C. (1998). Mechanistic study of disproportionate Permeability reduction. Presented at the 1998 SPE/DOE improved oil recovery Symposium held in Tulsa, Oklahoma. 1-10.

Niño L., de Sousa F. V. H. y Rosangela M. (2019). Less-Concentrated HPAM solutions as a Polymer Retention Reduction Method in CEOR. Revista Fuentes: El Reventón Energético, volumen 17 ( ${ }^{\circ}$ 2). 75-92.

Oghena A. (2012). Pressure Drawdown decline curve analysis. North Africa technical conference and exhibition held in Cairo, Egypt. 1-3.

Ortega A. (2013). Estudio del comportamiento de la inyección del sistema multigel ${ }^{\circledR}$ sellante en un pozo horizontal de la faja petrolífera del Orinoco para controlar la producción excesiva de agua. Universidad Central de Venezuela, para optar por el título de Ingeniero de Petróleos. 1-156.

Ortega A. T., Marin D. F. y Ochoa E. D. (2019). Revisión general de la producción elevada de agua en la industria del petróleo. Revista Fuentes: El Reventón Energético, volumen 17 ( $\mathrm{n}^{\circ}$ 2). 39-50

Paris de Ferrer M. (2009). Fundamentos de Ingeniería de Yacimientos. Ediciones Astro Data S. A. Maracaibo, Venezuela. $1-588$.

Paris de Ferrer M. (2001). Inyección de agua y gas en yacimientos petrolíferos. Ediciones Astro Data S. A. Maracaibo, Venezuela. $1-390$.

Presidencia de la Republica de Colombia (2015). Resolución 0631. 1-93.

Peirce J. W., Hutcherson M. R., Jensen M. D., Brice B. W., Vasquez J. E., y Woods A. (2014). An 
Overview of Conformance Control Efforts for the West Sak Field on the North Slope of Alaska. SPE Improved Oil Recovery Symposium held in Tulsa, Oklahoma, USA. 1-20.

Ramos L. y Marín A. (2018). Evaluación y experiencias en el control de conificación en pozos con alto corte de agua en yacimientos con empuje hidráulico. Revista Fuentes: El Reventón Energético, volumen 16 ( $\left.\mathrm{n}^{\circ} 2\right)$. 24-40.

R.S. Seright, Fan T., Wavrik K. y de Carvalho Balaban R. (2010). New Insights into polymer Rheology in Porous Media. SPE Improved Oil Recovery Symposium held in Tulsa, Oklahoma. 1-11.

Romero J.L., (2018). Waterflood conformance improvement-practical considerations and lessons learned. Fuentes: el Reventón Energético, volumen 16 (no. 2). 7-21.

Romero L., Manalo F. y Kantzas A. (2008). Characterization of Crosslinked Gel kinetics and gel strength by use of NMR. SPE Reservoir Evaluation and Engineering. 1-15.

Salgaonkar L. y Das. (2012). Laboratory Evaluation of organically crosslinked polymer for water shutoff in high-temperature wells applications. SPE Kuwait international petroleum conference and exhibition held in Kuwait City, Kuwait. 1-8.

Sampaio M.A., Montoya S.D. y González J.C. (2018). Production optimization for a conceptual model through combined use of polymer flooding and intelligent well technology under uncertainties. Fuentes: El Reventón energético, volumen $16\left(\mathrm{n}^{\circ}\right.$ 1). $37-45$.

Simjoo M., Koohi A.D., Vafaie-Sefti M. y Zitha P.L.J. (2009). Water shut-off in a fractured system using a robust polymer gel. SPE European formation damage conference held in Scheveningen, The Netherlands. 1-8.

Sparlin D.D. (1976). An Evaluation of polyacrylamides for reducing water production. Journal of Petroleum Technology. 1-12.

Stavland A., Andersen L.I., Standoy B., Tjomsland T. y Metrabu A.A. (2006). How to apply a Blocking gel system for bullhead selective water shutoff: from laboratory to field. SPE/DOE Symposium on Improved Oil Recovery held in Tulsa, Oklahoma. $1-12$.

Sun Y., Fang Y., Chen A., You Q., Dai C., Cheng R. y Liu Y. (2017). Gelation behavior study of a Resorcinol-Hexamethyleneteramine crosslinked polymer gel for water shutoff treatment in low temperature and high salinity reservoirs. Energies. $1-14$.

Syndansk R.D. (1988). A New ConformanceImprovement-Treatment Chromium (III) Gel Technology. SPE/DOE enhanced oil recovery symposium held in Tulsa, Oklahoma. 1-16.

Syndansk R.D. y Seright R.S. (2006). When and where relative permeability modification watershutoff treatments can be successfully applied. $\mathrm{SPE} / \mathrm{DOE}$ symposium on improved oil recovery held in Tulsa, Oklahoma. 1-15.

Taha A. y Amani M. (2019). Overview of water shutoff operations in Oil and Gas wells, Chemical and mechanical solutions. 1-11.

Tapias F.A., Lizcano J.C. y Lopes R.B.Z. (2018). Effects of salts and temperature on rheological and viscoelastic behavior of low molecular wight HPAM solutions. Fuentes: el Reventón Energético volumen 16 (no. 1). 19-35.

Thomas F.B., Bennion D.B., Anderson G.E., Meldrum B.T. y Heaven W.J. (2000). Water shut-off treatments-reduce water and accelerate oil production. JCPT volume $39, \mathrm{n}^{\circ}$ 4. 1-5.

van der Hoek J.E., Botermans W. y Zitha. L.J. (2001). Full blocking mechanism of polymer gels for water control. SPE European Formation Damage Conference held in The Hague, The Netherlands. $1-15$.

Vásquez J. y Eoff L. (2013). A Relative Permeability Modifier for Water Control: Candidate Selection, Case Histories, and Lessons Learned after more than 3,000 Well Interventions. SPE European Formation Damage Conference and Exhibition held in Noordwijk, The Netherlands. 1-13.

Vásquez J. y Rodríguez L. (2015). A water swelling polymer for fracture shutoff to improve injection profile during secondary recover: case histories 
and lessons learned after more than 600 well interventions. EUROPEC held in Madrid. 1-13.

Villegas I.A. (2013). Study of Technical feasibility for water control in the "U" inferior reservoir in the Pindo Oilfield. Universidad Central del Ecuador, Facultad de Ingeniería en Geología, Minas, Petróleos y Ambiental. 1-197.

Whittington L.E., D.G. Naae y R.W. Braun (1994). Brine-Initiated Gels: A new water Shut-Off Agent. Presented at the SPE/DOE ninth Symposium on improved Oil Recovery held in Tulsa, Oklahoma. $1-10$.

Xindi S. y Bai B. (2017). comprehensive review of water shutoff methods for horizontal wells. Petroleum exploration and development. 1-8.

Zaitoun A., Kohler N., Bossie-Codreanu y Denys K. (1999). Water Shutoff by relative permeability modifiers: lesson from several field applications. SPE annual technical conference and exhibition held in Houston, Texas. 1-14.

Zhu D., Bai B. y Hou J. (2017). Polymer Gel Systems for Water Management in High-Temperature Petroleum Reservoirs: A Chemical review. Energy fuels. 1-25.

Fecha de recepción: Octubre 3 de 2019

Fecha de aceptación: Diciembre 7 de 2020 